\title{
Genetic diversity and population structure of wild Dipsacus asperoides in China as indicated by ISSR markers
}

\author{
D.X. Chen ${ }^{1,2,3 *}$, L.Y. Li ${ }^{2,3 *}$, X. Zhang, ${ }^{2,3}$, Y. Wang ${ }^{2,3}$ and Z. Zhang ${ }^{1}$ \\ ${ }^{1}$ College of Life Sciences, Chongqing University, Chongqing, China \\ ${ }^{2}$ Chongqing Academy of Chinese Materia Medica, Chongqing, China \\ ${ }^{3}$ Chongqing Engineering Research Center for Fine Variety Breeding Techniques \\ of Chinese Materia Medica, Chongqing, China \\ *These authors contributed equally to this study. \\ Corresponding author: Z. Zhang \\ E-mail: zezhang@cqu.edu.cn
}

Genet. Mol. Res. 13 (3): 6340-6349 (2014)

Received June 12, 2013

Accepted October 3, 2013

Published February 14, 2014

DOI http://dx.doi.org/10.4238/2014.February.14.12

\begin{abstract}
In order to evaluate the genetic diversity and genetic structure of wild Dipsacus asperoides, we surveyed genetic polymorphisms in 288 individuals from 12 populations using ISSR. A total of 240 bands were amplified, among which 190 were polymorphic loci. At the species level, genetic diversity was found to be abundant: $\mathrm{PPB}=79.17 \%, N_{\mathrm{E}}=1.2152, H=0.1361$, and $H_{\mathrm{sp}}=0.2213$. At the population level, genetic diversity was lower: $\mathrm{PPB}=30.76 \%, N_{\mathrm{E}}=$ $1.0786, H=0.0897$, and $H_{\text {pop }}=0.1375$. The calculated Nei genetic differentiation coefficient was 0.3406 , which is consistent with the calculated Shannon population genetic differentiation coefficient of 0.3787 . This is commonly taken to indicate that most of the genetic variation existed within the populations. Gene flow was calculated as $N_{\mathrm{m}}=0.9679$, suggesting that gene exchange only occurred at a low level. Based on the Nei genetic distance, the 12 populations were divided into 4 categories. Our results suggest that $D$. asperoides has
\end{abstract}


abundant genetic diversity and provides a foundation for the protection and improvement of germplasm resources.

Key words: Dipsacus asperoides; Inter-simple sequence repeat; Genetic diversity; Genetic structure

\section{INTRODUCTION}

Dipsacus asperoides is a perennial herb of the Dipsacaceae family that is used in traditional medicine for its dried roots (Editorial Committee of Flora of China \& Chinese Academy of Sciences, 1986). In China, this plant is widely distributed throughout the provinces south of the Yangtze River, in the Gansu Province and in the southern Shanxi Province (Chen and $\mathrm{Ai}, 1997)$. It grows in shrubs, herbosa, and along roadsides. Because the domestic and foreign demand for the herb increases annually, the price of $D$. asperoides herbs has been rising. Because there is currently no large-scale artificial cultivation method for $D$. asperoides, most of the medicinal herbs come from wild resources. Therefore, $D$. asperoides had been harvested excessively, regardless of plant size or growth stage. This unscientific harvesting has weakened the natural reproductive capacity of the wild population. Furthermore, in the areas with greater human activity, the original habitat of $D$. asperoides has become over-grazed or been converted to farmland. As D. asperoides has suffered from substantial habitat deterioration, the number and size of wild populations of $D$. asperoides have decreased, resulting in the rapid shrinkage of the genetic diversity of the species. In recent years, with the sharp decline of $D$. asperoides wild resources, it has been gradually realized that the protection and rational utilization of resources has considerable importance. In order to protect and rationally utilize these resources, it is necessary to study the genetic diversity and genetic structure of populations of this plant.

ISSR (inter-simple sequence repeat), combined with the advantages of SSR and RAPD technology (Zietkiewicz et al., 1994), has been used successfully in the study of plants for traditional Chinese medicine (Shen et al., 2006; Zhang et al., 2010; Song et al., 2010; Wei and $\mathrm{Wu}, 2012)$. Most previous studies of $D$. asperoides have focused on germplasm resources (Chen and Ai, 1997), traditional morphological classifications (Li et al., 1993; Feng et al., 2000), chemicals (Liu et al., 2011), and pharmacological applications (Jung et al., 2012). Basic studies of the genetic structure of different populations using molecular markers have not yet been reported. This study is the first to use ISSR to survey the genetic structure and intraspecific genetic polymorphisms of 12 populations of this plant. Our results provide useful information for the selection of genetic breeding materials and a scientific basis for the development of protection strategies.

\section{MATERIAL AND METHODS}

\section{Plant materials}

In this study, 288 individuals from 12 wild $D$. asperoides populations were collected from the main distribution regions in China (sampling locations and the number of samples are shown in Figure 1 and Table 1). Sampling was performed in November 2010. Species identifi- 
cation was performed based on The Flora of China (Editorial Committee of Flora of China \& Chinese Academy of Sciences, 1986). According to the principle of uniform distribution and random sampling, 24 individuals from each population were sampled in an attempt to cover all of its distribution range and to collect individuals of different genotypes. Fresh young leaves from each sample plant were collected, washed, and air-dried at room temperature. They were next placed in a zip-lock bag containing silica gel to facilitate quick drying. For each population, the altitude, longitude, latitude, and other environmental factors were recorded using a GPS-positioning system (Table 1).

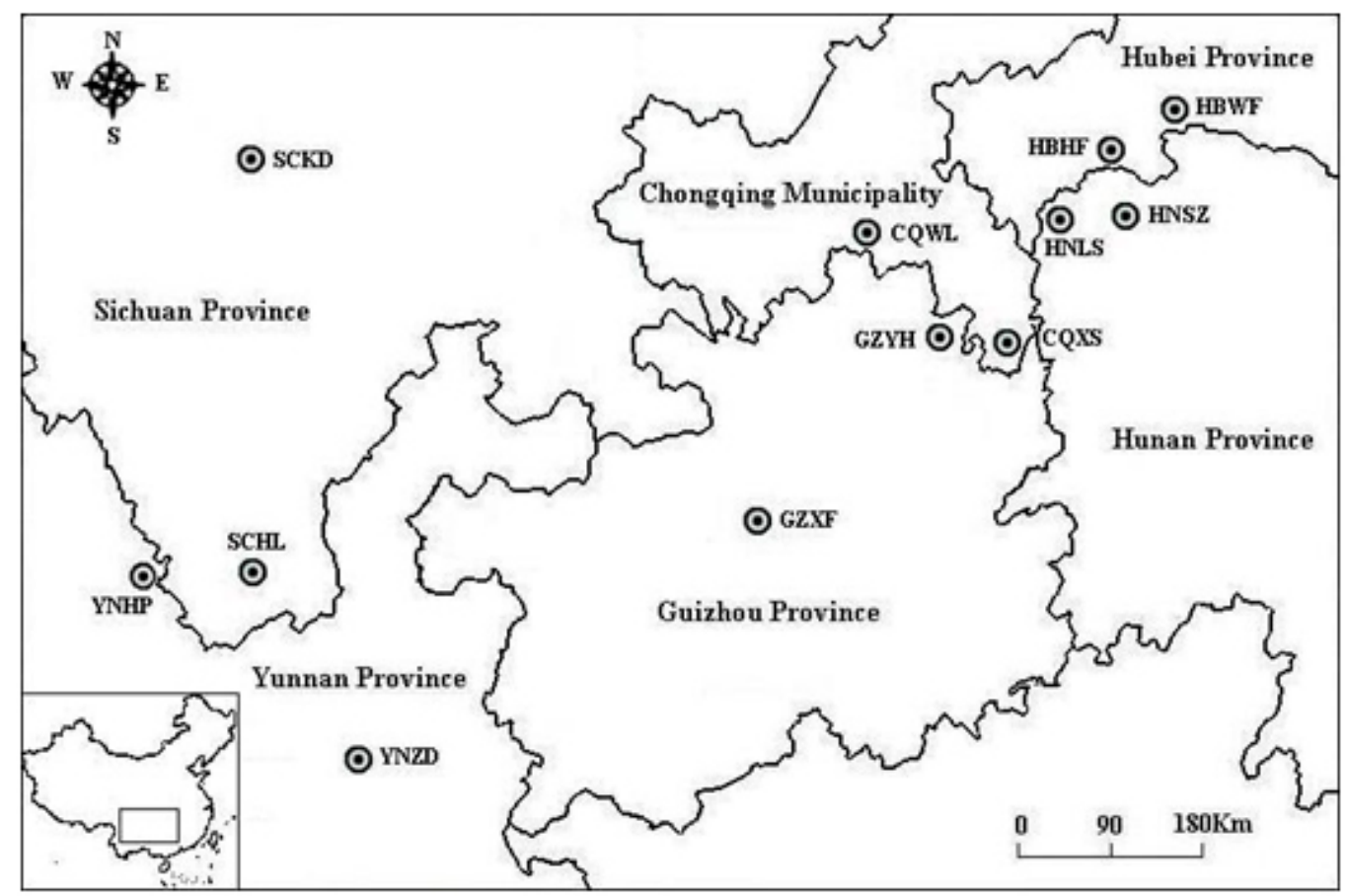

Figure 1. Map of the sampling localities in this study. The abbreviations and coordinates are listed in Table 1.

Table 1. Materials used in the study.
\begin{tabular}{llcccc}
\hline Population & Locality & Simple size & Altitude (m) & Longitude E & Latitude N \\
\hline CQWL & Wulong, Chongqing & 24 & 1320 & $107^{\circ} 24^{\prime} 64^{\prime \prime}$ & $29^{\circ} 24^{\prime} 65^{\prime \prime}$ \\
CQXS & Xioushan, Chongqing & 24 & 1070 & $108^{\circ} 59^{\prime} 66^{\prime \prime}$ & $28^{\circ} 28^{\prime} 54^{\prime \prime}$ \\
SCKD & Kangding, Sichuan & 24 & 2700 & $101^{\circ} 57^{\prime} 33^{\prime \prime}$ & $30^{\circ} 01^{\prime} 62^{\prime \prime}$ \\
SCHL & Huili, Sichuan & 24 & 2095 & $102^{\circ} 16^{\prime} 16^{\prime \prime}$ & $26^{\circ} 48^{\prime} 11^{\prime \prime}$ \\
YNZD & Zhongdian, Yunnan & 24 & 3270 & $99^{\circ} 40^{\prime} 18^{\prime \prime}$ & $27^{\circ} 52^{\prime} 63^{\prime \prime}$ \\
YNHP & Huaping, Yunnan & 24 & 1960 & $101^{\circ} 09^{\prime} 39^{\prime \prime}$ & $26^{\circ} 27^{\prime} 43^{\prime \prime}$ \\
GZYH & Yanhe, Guizhou & 24 & 950 & $108^{\circ} 25^{\prime} 00^{\prime \prime}$ & $28^{\circ} 25^{\prime} 43^{\prime \prime}$ \\
GZXF & Xifeng, Guizhou & 24 & 960 & $106^{\circ} 45^{\prime} 47^{\prime \prime}$ & $27^{\circ} 11^{\prime} 01^{\prime \prime}$ \\
HNLS & Longshan, Hunan & 24 & $109^{\circ} 40^{\prime} 40^{\prime \prime}$ & $29^{\circ} 26^{\prime} 52^{\prime \prime}$ \\
HNSZ & Sangzhi, Hunan & 24 & $109^{\circ} 54^{\prime} 09^{\prime \prime}$ & $29^{\circ} 27^{\prime} 11^{\prime \prime}$ \\
HBWF & Wufeng, Hubei & 24 & $110^{\circ} 34^{\prime} 24^{\prime \prime}$ & $29^{\circ} 49^{\prime} 40^{\prime \prime}$ \\
HBHF & Hefeng, Hubei & 24 & 1370 & $110^{\circ} 25^{\prime} 31^{\prime \prime}$ & $29^{\circ} 59^{\prime} 13^{\prime \prime}$ \\
\hline
\end{tabular}




\section{Extraction of genomic DNA}

Genomic DNA was extracted using a Tian Gen genomic DNA extraction kit. DNA quality and quantity were measured using a Smart $\mathrm{Spe}^{\mathrm{TM}} 3000$ Spectrophotometer (BIORAD; Hercules, CA, USA) and 1\% agarose gel electrophoresis, respectively.

\section{ISSR-PCR amplification and detection}

Using sequences published by the University of British Columbia (UBC, Vancouver, Canada), ISSR primers were synthesized by the Shanghai Sangon Biological Engineering Co., Ltd. We screened 40 ISSR primers in two randomly selected DNA templates from each population. Ultimately, we found 20 primers that yielded amplification products that were clear and reproducible, and showed relatively high polymorphisms that we used for formal amplification. Denaturation temperatures varied from $1^{\circ}$ to $3^{\circ} \mathrm{C}$. The names and sequences of the specific primers are shown in Table 2.

The ISSR reaction cocktail was designed as described in the literature (Chen et al., 2006). Briefly, a total volume of $25 \mu \mathrm{L}$ contained $1 \mathrm{X}$ PCR buffer, $\mathrm{Mg}^{2+}(1.5 \mathrm{mM})$, dNTPs (200 $\mu \mathrm{M})$, primers $(0.4 \mu \mathrm{M})$, genomic DNA (40 $\mathrm{ng})$, and Taq DNA polymerase (1 U). The amplification program was set for $94^{\circ} \mathrm{C}$ for $5 \mathrm{~min}$, and then 35 cycles of $94^{\circ} \mathrm{C}$ (denaturation) for $30 \mathrm{~s}$, $53^{\circ}-57^{\circ} \mathrm{C}$ (annealing) for $1 \mathrm{~min}$, and $72^{\circ} \mathrm{C}$ (extension) for $1.5 \mathrm{~min}$, and finishing with 1 cycle at $72^{\circ} \mathrm{C}$ for $7 \mathrm{~min}$, followed by storage at $4^{\circ} \mathrm{C}$. All reactions were carried out in a $\mathrm{S} 1000^{\mathrm{TM}}$ Thermal Cycler (BIO-RAD).

Using $1 \mathrm{X}$ TAE buffer, the amplified products were stained and separated by $1.5 \%$ agarose gel electrophoresis $(150 \mathrm{~V})$. When the bromophenol blue indicator was $2-3 \mathrm{~cm}$ from the edge of the agarose gel, electrophoresis was stopped. The gel products were visualized and recorded using an automated imaging system (Gel Doc XR; BIO-RAD).

\section{Statistical analysis}

Because ISSR is a dominant marker, amplified bands of the same primer showing the same electrophoretic mobility indicate homology. According to presence or absence of bands, the electrophoresis results were translated into a binary data matrix (i.e., for the same allele, the presence of a band was recorded as " 1 " and its absence was recorded as " 0 "). Bands that were ambiguous or difficult to measure were not included in statistical analysis. Among polymorphic loci, only stable differential bands were used for data analysis in the repeated trials.

We assumed that the populations used in this study were in Hardy-Weinberg equilibrium. The results of the amplification were analyzed for genetic parameters using the POPGENE 1.31 software. These parameters included: total gene diversity $\left(H_{\mathrm{T}}\right)$, gene diversity within populations $\left(H_{\mathrm{S}}\right)$, genetic differentiation coefficient $\left(G_{\mathrm{ST}}\right)$, gene flow $\left(N_{\mathrm{m}}\right)$, the Nei genetic distance $\left(D_{\mathrm{ST}}\right)$, genetic identity (I), the percentage of polymorphic bands (PPB), the Nei gene diversity $(H)$, the observed number of alleles $\left(N_{\mathrm{A}}\right)$, the effective number of alleles $\left(N_{\mathrm{E}}\right)$, and the Shannon information index $\left(H_{\mathrm{O}}\right)$. We calculated $H_{\mathrm{O}}$ at two levels, the total diversity $\left(H_{\mathrm{sp}}\right.$, species level) and the average diversity within a population $\left(H_{\mathrm{pop}}\right.$, population level). We estimated the genetic variation among populations using the Shannon population differentiation factor $\left(\left(H_{\mathrm{sp}}-H_{\mathrm{pop}}\right) / H_{\mathrm{sp}}\right)$, which is similar to the $G_{\mathrm{ST}}$ parameter. A dendrogram was generated using UPGMA clustering in order to analyze the genetic relationships among groups. 


\section{RESULTS}

\section{Amplification results generating using ISSR primers}

We used 20 ISSR primers that yielded clearly amplified bands and polymorphic bands, and detected amplification in all 288 samples (Table 2). The number of amplified bands varied with the different primers. Each primer yielded 7-18 bands and 4-16 polymorphic bands with an average of 12.0 bands in total and 9.5 polymorphic bands per primer. A total of 240 bands were amplified, 190 of which were polymorphic (Table 2). The rate of polymorphism differed markedly among primers, ranging from 55.6 to $100.0 \%$, and the level of polymorphism for most primers was moderately high. This showed that the efficiency of ISSR markers was high with respect to the detection of polymorphisms in the analysis of the genetic diversity of $D$. asperoides.

\begin{tabular}{|c|c|c|c|c|}
\hline Primer & Sequence & Total bands & Polymorphic bands & Percentage of polymorphic bands \\
\hline UBC 808 & $(\mathrm{AG})_{8} \mathrm{C}$ & 16 & 11 & 68.75 \\
\hline UBC809 & $(\mathrm{AG})_{8}^{8} \mathrm{G}$ & 11 & 9 & 81.82 \\
\hline UBC810 & $(\mathrm{GA})_{8} \mathrm{~T}$ & 16 & 15 & 93.75 \\
\hline UBC811 & $(\mathrm{GA})_{8}^{8} \mathrm{C}$ & 17 & 13 & 76.47 \\
\hline UBC812 & $(\mathrm{GA})_{8} \mathrm{~A}$ & 8 & 6 & 75.00 \\
\hline UBC815 & $(\mathrm{CT})_{8}^{8} \mathrm{G}$ & 18 & 16 & 88.89 \\
\hline UBC818 & $(\mathrm{CA})_{8}^{8} \mathrm{G}$ & 15 & 15 & 100.00 \\
\hline UBC822 & $(\mathrm{TC})_{8}^{8} \mathrm{~A}$ & 14 & 11 & 78.57 \\
\hline UBC824 & $(\mathrm{TC})_{8}^{8} \mathrm{G}$ & 11 & 11 & 100.00 \\
\hline UBC 825 & $(\mathrm{AC})_{8} \mathrm{~T}$ & 11 & 10 & 90.91 \\
\hline UBC 828 & $(\mathrm{TG})_{8}^{8} \mathrm{~A}$ & 10 & 8 & 80.00 \\
\hline UBC836 & $(\mathrm{AG})_{8} \mathrm{YA}$ & 9 & 6 & 66.67 \\
\hline UBC841 & $(\mathrm{GA})_{8}^{8} \mathrm{YC}$ & 10 & 7 & 70.00 \\
\hline UBC844 & $(\mathrm{CT})_{8}^{8} \mathrm{RC}$ & 16 & 13 & 81.25 \\
\hline UBC 848 & $(\mathrm{CA})_{8}^{8} \mathrm{RG}$ & 7 & 4 & 57.14 \\
\hline UBC 855 & $(\mathrm{AC})_{8}^{8} \mathrm{YT}$ & 12 & 8 & 66.67 \\
\hline UBC857 & $(\mathrm{AC})_{8} \mathrm{YG}$ & 13 & 9 & 69.23 \\
\hline UBC868 & $(\mathrm{GAA})_{6}$ & 9 & 8 & 88.89 \\
\hline UBC 881 & $\left(\mathrm{GGGTG}_{3}\right.$ & 9 & 5 & 55.56 \\
\hline UBC891 & $\mathrm{HVH}(\mathrm{TG})_{7}^{3}$ & 8 & 5 & 62.50 \\
\hline Total & & 240 & 190 & 79.17 \\
\hline
\end{tabular}

$\mathrm{R}=(\mathrm{A}, \mathrm{G}) ; \mathrm{Y}=(\mathrm{C}, \mathrm{T}) ; \mathrm{H}=(\mathrm{A}, \mathrm{C}, \mathrm{T})$ (i.e., $\operatorname{not} \mathrm{G}) ; \mathrm{V}=(\mathrm{A}, \mathrm{C}, \mathrm{G})$ (i.e., $\operatorname{not} \mathrm{T})$.

\section{Genetic diversity in 12 populations of $D$. asperoides}

Using the POPGENE software, we determined the associated genetic parameters at the species and population levels (Table 3 ). At the species level, $\mathrm{PPB}=79.17 \%, N_{\mathrm{E}}=1.2152$, $H=0.1361$, and $H_{\mathrm{sp}}=0.2213$, which showed that $D$. asperoides has abundant genetic diversity. At the population level, the average and range of genetic parameters in various populations were found to be $\mathrm{PPB}=30.8 \%(25.0-33.3 \%), N_{\mathrm{E}}=1.0786(1.1242-1.1693), H=0.0897$ (0.0735-0.1012), and $H_{\text {pop }}=0.1375$ (0.1116-0.1543), showing that the level of genetic diversity among the 12 populations was low and that the differences were not significant.

\section{Population genetic structure in $D$. asperoides}

The parameters used to measure the genetic structure of the population include $H_{\mathrm{T}}, H_{\mathrm{S}}$, $D_{\mathrm{ST}}=H_{\mathrm{T}}-H_{\mathrm{S}}, G_{\mathrm{ST}}$, and $N_{\mathrm{m}}$. 
The Nei gene diversity was estimated as $H_{\mathrm{T}}=0.1361, H_{\mathrm{S}}=0.0897$, and $D_{\mathrm{ST}}=0.0464$. The Nei gene differentiation coefficient, $G_{\mathrm{ST}}=0.3406$, showed that the total genetic variation came less from variation among populations $(34.1 \%)$ and more from variation within populations $(65.9 \%)$. This shows that genetic differentiation within populations is much more pronounced than differentiation among populations and that variation within populations is the main source of variation in $D$. asperoides.

Table 3. Genetic variation among Dipsacus asperoides populations.

\begin{tabular}{lccccccc}
\hline Population & $\mathrm{N}$ & $\mathrm{K}$ & $\mathrm{PPB}$ & $N_{\mathrm{A}}(\mathrm{SD})$ & $N_{\mathrm{E}}(\mathrm{SD})$ & $H(\mathrm{SD})$ & $H_{\mathrm{O}}(\mathrm{SD})$ \\
\hline CQWL & 24 & 70 & $29.17 \%$ & $1.2917(0.4555)$ & $1.1583(0.3057)$ & $0.0930(0.1677)$ & $0.1406(0.2435)$ \\
CQXS & 24 & 79 & $32.92 \%$ & $1.3292(0.4709)$ & $1.1693(0.3050)$ & $0.1012(0.1694)$ & $0.1543(0.2470)$ \\
SCKD & 24 & 60 & $25.00 \%$ & $1.2500(0.4339)$ & $1.1273(0.2894)$ & $0.0735(0.1554)$ & $0.1116(0.2236)$ \\
SCHL & 24 & 75 & $31.25 \%$ & $1.3125(0.4645)$ & $1.1599(0.2983)$ & $0.0958(0.1659)$ & $0.1462(0.2422)$ \\
YNZD & 24 & 80 & $33.33 \%$ & $1.3333(0.4724)$ & $1.1681(0.3061)$ & $0.1000(0.1696)$ & $0.1525(0.2464)$ \\
YNHP & 24 & 74 & $30.83 \%$ & $1.3083(0.4628)$ & $1.1430(0.2862)$ & $0.0864(0.1575)$ & $0.1336(0.2303)$ \\
GZYH & 24 & 80 & $33.33 \%$ & $1.3333(0.4724)$ & $1.1640(0.3055)$ & $0.0973(0.1682)$ & $0.1487(0.2439)$ \\
GZXF & 24 & 78 & $32.50 \%$ & $1.3250(0.4694)$ & $1.1504(0.2840)$ & $0.0919(0.1599)$ & $0.1421(0.2348)$ \\
HNLS & 24 & 79 & $32.92 \%$ & $1.3292(0.4709)$ & $1.1433(0.2750)$ & $0.0886(0.1551)$ & $0.1386(0.2284)$ \\
HNSZ & 24 & 77 & $32.08 \%$ & $1.3208(0.4678)$ & $1.1242(0.2587)$ & $0.0773(0.1464)$ & $0.1222(0.2157)$ \\
HBWF & 24 & 69 & $28.75 \%$ & $1.2875(0.4535)$ & $1.1461(0.2992)$ & $0.0854(0.1632)$ & $0.1296(0.2359)$ \\
HBHF & 24 & 65 & $27.08 \%$ & $1.2708(0.4453)$ & $1.1447(0.2898)$ & $0.0860(0.1619)$ & $0.1302(0.2363)$ \\
Average & & 74 & $30.76 \%$ & $1.3076(0.4613)$ & $1.0786(0.2919)$ & $0.0897(0.1610)$ & $0.1375(0.2357)$ \\
Species level & 28 & 190 & $79.17 \%$ & $1.7917(0.4070)$ & $1.2152(0.3060)$ & $0.1361(0.1660)$ & $0.2213(0.2356)$
\end{tabular}

$\mathrm{N}=$ sample sizes; $\mathrm{K}=$ polymorphic loci; $\mathrm{PPB}=$ percentage of polymorphic bands; $N_{\mathrm{A}}=$ observed number of alleles; $N_{\mathrm{E}}=$ effective number of alleles; $H=$ Nei's gene diversity; $H_{\mathrm{O}}=$ Shannon's information index $\left(H_{\text {pop }}=\right.$ population level, $H_{\mathrm{sp}}=$ species level).

Shannon's diversity index was used to measure diversity at the species level $H_{\mathrm{sp}}=$ 0.2213 and population level $H_{\text {pop }}=0.1375$ (Table 3). Based on these data, we calculated the Shannon population differentiation coefficient $\left(H_{\mathrm{sp}}-H_{\mathrm{pop}}\right) / H_{\mathrm{sp}}$ to be 0.3787 , showing that $37.9 \%$ of the genetic variation was distributed among populations and $62.1 \%$ was found within populations.

The results of analysis of these two types of methods were nearly identical. Only small differences were observed, showing that the genetic differentiation was not obvious among the populations and that most of the genetic variation existed within the populations.

Gene flow can be considered as the number of individuals who migrated from one population to another per generation, indicating the extent of genetic exchange among populations. It can be calculated based on the following formula: $N_{\mathrm{m}}=0.5\left(1-G_{\mathrm{ST}}\right) / G_{\mathrm{ST}}$. Wright (1951) reported that $N_{\mathrm{m}}>1$ indicates gene flow between populations. Gene flow among populations in $D$. asperoides was 0.9679 , which is less than 1 , indicating a low level of genetic exchange and a high degree of genetic differentiation.

\section{Genetic identity and genetic distance among $D$. asperoides populations}

In order to assess the genetic relationship among $D$. asperoides populations, we calculated the genetic identity and genetic distance using POPGENE (Table 4). Our results showed that the Nei genetic identity ranged from 0.8981 to 0.9858 , and the genetic distance ranged from 0.0143 to 0.1075 . Genetic identity was the lowest between POP3 and POP9 ( $\mathrm{I}=0.8981$ ), but the genetic distance between these two populations was the longest $\left(D_{\mathrm{ST}}=0.1075\right)$, showing 
the most distant phylogenetic relationship between these two populations. Genetic identity was the greatest between POP11 and POP12 ( $\mathrm{I}=0.9858)$, and their genetic distance was the shortest $(\mathrm{D}=0.0143)$, indicating the closest phylogenetic relationship between these two populations.

Table 4. Genetic identity (above diagonal) and genetic distance (below diagonal) among Dipsacus asperoides populations.

\begin{tabular}{lcccccccccccc}
\hline & POP1 & POP2 & POP3 & POP4 & POP5 & POP6 & POP7 & POP8 & POP9 & POP10 & POP11 & POP12 \\
\hline POP1 & $* * * *$ & 0.9806 & 0.9068 & 0.9522 & 0.9099 & 0.9306 & 0.9549 & 0.9524 & 0.9548 & 0.9478 & 0.9495 & 0.9461 \\
POP2 & 0.0196 & $* * * *$ & 0.9169 & 0.9605 & 0.9183 & 0.9369 & 0.9624 & 0.9549 & 0.9493 & 0.9431 & 0.9486 & 0.9436 \\
POP3 & 0.0978 & 0.0868 & $* * * *$ & 0.9450 & 0.9171 & 0.9144 & 0.9185 & 0.9121 & 0.8981 & 0.9012 & 0.9113 & 0.9082 \\
POP4 & 0.0490 & 0.0403 & 0.0565 & $* * * *$ & 0.9347 & 0.9603 & 0.9571 & 0.9570 & 0.9454 & 0.9460 & 0.9553 & 0.9512 \\
POP5 & 0.0944 & 0.0852 & 0.0866 & 0.0675 & $* * * *$ & 0.9429 & 0.9318 & 0.9243 & 0.9159 & 0.9160 & 0.9219 & 0.9199 \\
POP6 & 0.0719 & 0.0652 & 0.0895 & 0.0405 & 0.0588 & $* * * *$ & 0.9484 & 0.9515 & 0.9370 & 0.9368 & 0.9413 & 0.9347 \\
POP7 & 0.0462 & 0.0384 & 0.0850 & 0.0438 & 0.0706 & 0.0530 & $* * * *$ & 0.9718 & 0.9619 & 0.9595 & 0.9634 & 0.9636 \\
POP8 & 0.0488 & 0.0461 & 0.0920 & 0.0440 & 0.0787 & 0.0497 & 0.0287 & $* * * *$ & 0.9617 & 0.9613 & 0.9637 & 0.9682 \\
POP9 & 0.0462 & 0.0521 & 0.1075 & 0.0562 & 0.0878 & 0.0651 & 0.0388 & 0.0391 & $* * * *$ & 0.9833 & 0.9824 & 0.9762 \\
POP10 & 0.0537 & 0.0586 & 0.1040 & 0.0555 & 0.0877 & 0.0653 & 0.0413 & 0.0394 & 0.0168 & $* * * *$ & 0.9819 & 0.9774 \\
POP11 & 0.0519 & 0.0527 & 0.0928 & 0.0458 & 0.0813 & 0.0605 & 0.0373 & 0.0369 & 0.0177 & 0.0182 & $* * * *$ & 0.9858 \\
POP12 & 0.0554 & 0.0581 & 0.0963 & 0.0500 & 0.0835 & 0.0675 & 0.0370 & 0.0323 & 0.0241 & 0.0229 & 0.0143 & $* * * *$ \\
\hline
\end{tabular}

\section{Cluster analysis of $D$. asperoides populations}

Based on the Nei genetic distance, a dendrogram showing the phylogenetic relationships was constructed using UPGMA methods (Figure 2). Cluster analysis showed that the 12 populations clustered into 4 categories. The first category consisted of 8 populations from Chongqing, Guizhou, Hunan, and Hubei Provinces, the second category included the SCHL population from Sichuan and the YNHP population from Yunnan, the third category included only the YNZD population, and the fourth category included only the SCKD population. As can be seen from the clustering results, the geographic distribution of the 12 populations and the germplasm whose geographical distance was shorter tended to be in the same category. SPSS analysis indicated a moderate, significant correlation between the genetic and geographic distances among populations $(r=0.625, \mathrm{P}<0.01)$. This showed that the distribution of genetic variation among populations followed obvious geographic trends.

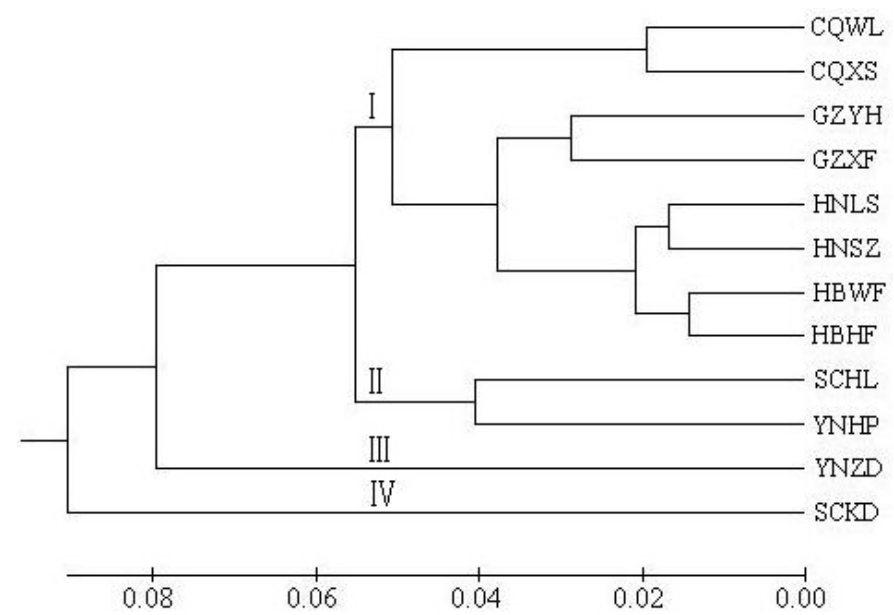

Figure 2. Dendrogram of twelve Dipsacus asperoides populations. 


\section{DISCUSSION}

\section{Relationships between wild $D$. asperoides populations}

Most studies suggest a correlation between the geographical distribution of germplasm and molecular markers (Zhang et al., 2012; Zhou et al., 2012). The results presented here confirmed the existence of a correlation between the genetic relationship of $D$. asperoides germplasm resources and geographic distribution. This was especially obvious in certain small branches of the population. This may be because the germplasm of plants that were separated by less geographic distance grew in similar ecological environments. The 8 populations in the first category were found at similar altitudes. In the second category, the SCHL population and the YNHP population are located in different administrative regions, but the actual distance between them was small. These two populations have similar ecological environments and grow at nearly the same altitude.

D. asperoides is capable of living in a variety of environments. Its wide distribution can create different environments, even within the same geographic area. Sichuan Province alone has both high mountain canyons and hummocky plateaus. This is why the 2 populations from Sichuan Province clustered in different categories and why the 2 populations from Yunnan Province were also in different categories.

The YNZD and SCKD populations separately clustered into one category, possibly because of their unique geographic and ecological environment. The YNZD population is found in an alpine meadow at $3270 \mathrm{~m}$ altitude and is associated with the D. chinensis, whereas SCKD population grows in alpine regions and is associated with the D. kangdingensis.

\section{Genetic diversity}

In this paper, our results were obtained using a variety of statistical methods that indicated more abundant genetic diversity in $12 \mathrm{D}$. asperoides wild populations ( $\mathrm{PPB}=$ $79.17 \%, N_{\mathrm{E}}=1.2152, H=0.1361$, and $H_{\mathrm{sp}}=0.2213$ ) and a high level of genetic variation. The reason for this may be related to genetic distribution and breeding capacity. In general, a widely distributed species can maintain more genetic variation than a narrowly distributed species (Hamrick and Godt, 1989). D. asperoides is a widely distributed species of the Dipsacaceae family. Within its broad geographic area, the complexity and diversity of the terrain and weather conditions are conducive to the maintenance of a high level of genetic variation at the species level. In addition, the reproductive capacity of a species is related to genetic diversity. Generally, a species with a strong reproductive ability has a high level of genetic diversity (Huh, 1999). D. asperoides is a perennial plant capable of flowering and producing seeds the following year. Biennial mature plants can produce more than 2000 seeds. As determined by laboratory studies, the current germination rate of wild $D$. asperoides seeds harvested can exceed $85 \%$. This shows a strong reproductive capacity sufficient to maintain high levels of genetic diversity in $D$. asperoides.

\section{Barriers to gene flow}

Plant gene flow among and within populations is achieved by means of carriers of genetic 
material on media such as pollen, seeds, spores, or vegetative elements (Hamrick, 1987). Of these, pollen and seed dispersal are the most important. For D. asperoides, barriers to gene flow may be related to limited communication among pollen and seeds and to geographical isolation effects. First, $D$. asperoides is an entomophilous flower, so the migration distance of its pollen is shorter and more focused, that is, the possibility of an external pollen (gene) moving into its vicinity is low. Second, in the field survey, we found that many seedlings grew densely under each mother plant. Under natural conditions, this confirmed that the main mode of transmission of $D$. asperoides seeds relies on gravity, so its propagation distance is quite short. Third, although its distribution area was large, the field survey showed a trend toward certain habitats. Most populations were scattered into island-like clusters and separated from each other. Sometimes a river or valley served as a clear dividing line between populations and individuals located in the area valley composed of an effective population. The scale of most populations was very small, limiting gene flow among populations, and causing significant genetic drift.

\section{Protection strategies of $D$. asperoides genetic diversity}

As indicated by the characteristics of population genetic variation in $D$. asperoides, the following actions may serve to protect this species and foster the rational utilization of natural resources: 1) in situ conservation: protection should be focused on populations with more genetic diversity, such as the YNZD and GZYH populations evaluated here; 2) ex situ conservation: as the genetic variation among $D$. asperoides populations involves significant differentiation and mainly derives from within populations, ex situ conservation must not only involve sampling from as many populations as possible, but also from individuals that are widely spaced within populations in order to prevent the collection of clones of the same plant; and 3) wild upbringing: as imitation of the original ecological environment of $D$. asperoides and artificial dispersal of its seeds among populations would enhance genetic exchange.

\section{ACKNOWLEDGMENTS}

Research supported by the National Science and Technology Support Program of China (\#2006BAI06A11-09), the Scientific and Technical Personnel Service Enterprises Action Items of the Ministry of Science and Technology in China (\#2009GJF10045) and Chongqing Municipal Health Bureau of Traditional Chinese Medicine Science and Technology Projects (\#2012-2-42).

\section{REFERENCES}

Chen DX, Li LY, Peng R and Qu XY (2006). Genetic diversity of Coptis chinensis germplasm based on ISSR analysis. Zhongguo Zhong Yao Za Zhi. 31: 1937-1940.

Chen H and Ai T (1997). Medicinal plant resources of Dipsacaceae in China. Zhongguo Zhong Yao Za Zhi 22: 649-52, 702. Editorial Committee of Flora of China \& Chinese Academy of Sciences (1986). The Flora of China. Science Press, Beijing. Feng XF, Ai TM and Xu HN (2000). A study on pollen morphology of Dipsacus. Zhongguo Zhong Yao Za Zhi 25: 394-401. Hamrick JL (1987). Gene Flow Distribution of Genetic Variation in Plant Populations. In: Differentiation Patterns in Higher Plants (Urbanska KM, ed.). Academic Press, New York, 53-67.

Hamrick JL and Godt MJW (1989). Allozyme Diversity in Plant Species, In: Plant Population Genetics, Breeding and Genetic Resources (Brown AHD, Clegg MT, Kahler AL and Weir BS, eds.). Sinauer Associates Inc., Sunderland, 43-63. 
Huh MK (1999). Genetic diversity and population structure of Korean alder (Alnus japonica; Betulaceae). Can. J. For. Res. 29: 1311-1316.

Jung HW, Jung JK, Son KH, Lee DH, et al. (2012). Inhibitory effects of the root extract of Dipsacus asperoides C.Y. Cheng et al T.M.Ai on collagen-induced arthritis in mice. J. Ethnopharmacol. 139: 98-103.

Li YL, Ai TM and Fu GF (1993). Studies on microscopic identification of Chinese herbal medicine Xuduan. China J. Chin. Mat. Med. 18: 265-268.

Liu JJ, Huang WH, Lv ML, Si JP, et al. (2011). Determination of ergosterol in Ganoderma lucidum from different varieties and cultured tree species by HPLC. Zhong Yao Cai 34: 187-190.

Shen J, Ding X, Liu D, Ding G, et al. (2006). Intersimple sequence repeats (ISSR) molecular fingerprinting markers for authenticating populations of Dendrobium officinale Kimura et Migo. Biol. Pharm. Bull. 29: 420-422.

Song Z, Li X, Wang H and Wang J (2010). Genetic diversity and population structure of Salvia miltiorrhiza Bge in China revealed by ISSR and SRAP. Genetica 138: 241-249.

Wei L and Wu XJ (2012). Genetic variation and population differentiation in a medical herb Houttuynia cordata in China revealed by inter-simple sequence repeats (ISSRs). Int. J. Mol. Sci. 13: 8159-8170.

Wright S (1951). The genetical structure of populations. Ann. Eugen. J. 15: 323-354.

Zhang F, Lv Y, Dong H and Guo S (2010). Analysis of genetic stability through intersimple sequence repeats molecular markers in micropropagated plantlets of Anoectochilus formosanus Hayata, a medicinal plant. Biol. Pharm. Bull. 33: 384-388.

Zhang WJ, Lin YS, Cao WQ and Yang J (2012). Genetic diversity and variance of Stentor coeruleus (Ciliophora: Heterotrichea) inferred from inter-simple sequence repeat (ISSR) fingerprinting. J. Eukaryot. Microbiol. 59: 157-162.

Zhou TH, Dong SS, Li S and Zhao GF (2012). Genetic structure within and among populations of Saruma henryi, an endangered plant endemic to China. Biochem. Genet. 50: 146-158.

Zietkiewicz E, Rafalski A and Labuda D (1994). Genome fingerprinting by simple sequence repeat (SSR)-anchored polymerase chain reaction amplification. Genomics 20: 176-183. 